\title{
Corrigendum: Inclusion flotation-driven channel segregation in solidifying steels
}

Dianzhong Li, Xing-Qiu Chen, Paixian Fu, Xiaoping Ma, Hongwei Liu, Yun Chen, Yanfei Cao, Yikun Luan \& Yiyi Li

Nature Communications 5:5572 doi: 10.1038/ncomms6572 (2014); Published 25 Nov 2014; Updated 9 Feb 2015

In this Article, Supplementary Movies 1 and 2 were incorrectly associated with experiments VII and VIII, respectively. Instead, Supplementary Movie 1 corresponds to experiment VI and Supplementary Movie 2 corresponds to experiment VII. 